\title{
PROGRAM FOR THE ANALYSIS OF PLANE TRUSSES
}

\author{
John K. Makunza \\ Lecturer in Structural Engineering \\ University of Dar es Salaam, Tanzania
}

\begin{abstract}
The 'TRAP - TRuss Analysis Program' can assist engineers in the analysis of plane truss-type structures, as well as prove an excellent basis for understanding the "finite - element" method. The Program is completely menu-driven, with all nodes, elements, and load data entered through an input file. TRAP includes plotting for displaying the truss geometry in its original shape showing all nodes and support points. In the analysis process, firstly the structure data; e.g number of nodes, elements, materials and supports are to be defined. Secondly, element properties and support constraints have to be specified. Finally, nodal global loads are then defined. The program output include; displacements for each node, axial forces, length of each element and reactions at the supports.

The program aims at reducing the time of analysis and increase the accuracy of calculations as well as storage of the analysed data. The program caters for manual methods of truss analysis such as method of sections or joint method. It has been found that the program is efficient and has no limitations on the number of nodes and elements. The program is suitable for use by design engineers in analysing Plane - Trusses of any size.
\end{abstract}

\subsection{INTRODUCTION}

The 'TRAP - TRuss Analysis Program' can assist designers in the analysis of plane trusses by speeding the analysis work and increasing the accuracy together with enhanced storage of the analyzed data. This will cater for manual analysis of trusses which is tedious, susceptible to errors, and takes longer time especially when the number of elements is large, like the truss shown in Figure 1.

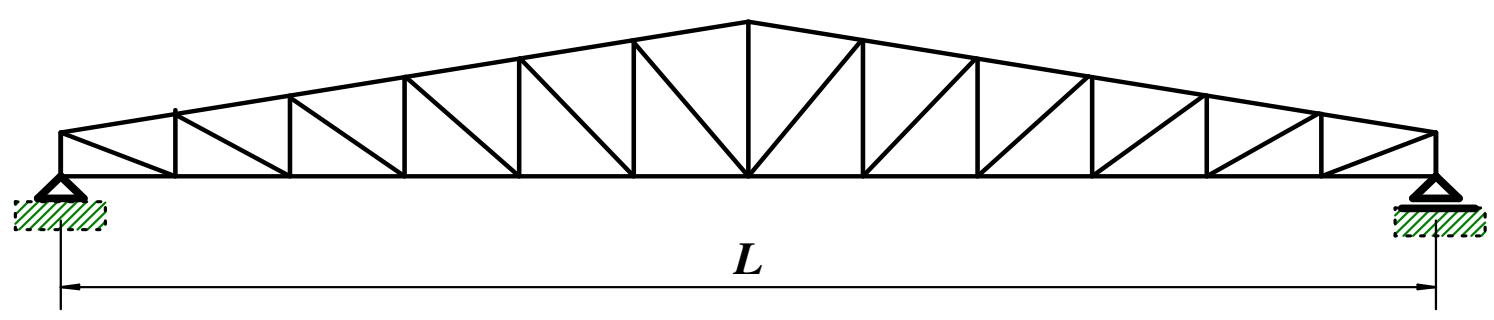

Figure 1: Plane truss

The program TRAP has been developed using the stiffness or displacement method of analysis $[1,2,3]$ because it is an effective tool in the analysis of both statically determinate and indeterminate structures. In additional to that, the method yields the displacements and forces directly, and, it is generally much easier to formulate the necessary matrices for the computer operations using the displacement method; and once this is done, the computer calculations can be performed efficiently. The program is suitable for use by design engineers in analysing Plane - Trusses of any size.

\subsection{THE STIFFNESS METHOD OF ANALYSIS}

Application of the stiffness method of analysis requires subdividing the structure into a series of discrete finite elements and identifying their end points as nodes. For truss analysis the finite elements are represented by each of the members that compose the truss, and the nodes represents the joints. The force-displacement properties of each element are determined and then related to one another using the force equilibrium equations written at the nodes. These relationships, for the entire structure, are then grouped together into what is called the structure stiffness matrix $\mathbf{K}$. Once it is established, the unknown displacements of the nodes can then be determined for any given loading on the structure. When these displacements are known, the external and internal forces in the structure can be calculated using the force displacement relations for each member. Some basic definitions and concepts of the stiffness method of analysis are herein given: 


\subsection{Identification of Nodes and Members}

The first step when applying the stiffness method is to identify the nodes as well as elements or members of the structure. For example in Figure 2(a), each node is identified by an inscribed number and each member is denoted by a plain number. Also, the "near" and "far" ends of the member are identified as shown in Figure 2(b). The tiny numbers show the two degrees of freedom at each node.

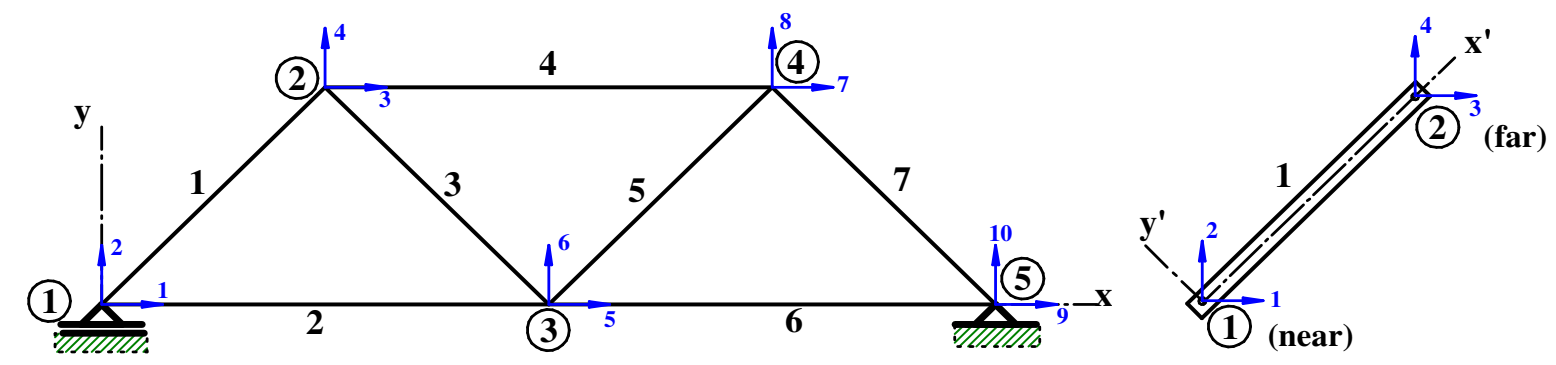

(a) Truss

(b) member 1

Figure 2: Identification of nodes, members and degrees of freedom

\subsubsection{Global and Member Coordinates}

In plane trusses, there are two coordinate systems, the first one is a single global or structure coordinate system, using $\mathrm{x}, \mathrm{y}$ axes, which is used to specify the sense of each of the external force and displacement components because they are vector quantities. The second is the local or member coordinate system identified using x', y' axes which is used for each member to specify the sense of direction of its displacements and internal actions. The origin is at the "near" node and the x' axis extends toward the "far" node as shown in Fig.2(b) for member 1.

\subsubsection{Degrees of Freedom}

Each node of a plane truss has two degrees of freedom; one in each global coordinate direction. The unconstrained degrees of freedom for the structure represent the primary unknowns in the stiffness method equations. For example, the truss in Figure 2 has 10 degrees of freedom, in which, at nodes 1 and 5 there are constrained degrees of freedom, while all others are unknown or unconstrained degrees of freedom. Due to the constraints, the displacements at node 1 ; in $\mathrm{y}-$ direction is zero and at node 5 both displacements are zero.

\subsection{Deformation of plane truss element}

Each plane truss element deforms axially along its axis. Axial deformation is the lengthening of a line element caused by equal and opposite longitudinal forces as shown in Figure 3.

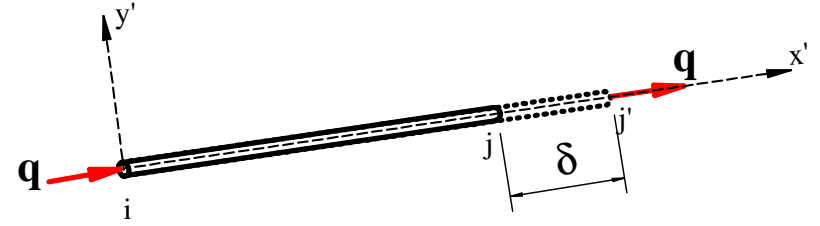

Figure 3: Axial deformation

The axial deformation of an element of length $\boldsymbol{L}$ is governed by equation:

$$
\delta=q \frac{L}{E A}
$$

where $\boldsymbol{\delta}=$ axial deformation

$\boldsymbol{q}=$ axial load

$\boldsymbol{L}=$ length of the element

$\boldsymbol{E}=$ elastic modulus (Young's modulus of elasticity)

$\boldsymbol{A}=$ cross section area of the element

From Equation (1), the axial force $\boldsymbol{q}$ can be expressed as:

$$
q=\frac{A E}{L} \delta
$$

The value $\frac{\boldsymbol{A} \boldsymbol{E}}{\boldsymbol{L}}$ is termed as stiffness, $\mathbf{k}$, and therefore Equation (2) may be rewritten as

$$
q=\boldsymbol{k} \delta
$$

\subsection{The Element Stiffness Matrix in Element Axes}

The forces at the ends of a truss member are related to the displacements at the ends by the element stiffness matrix. Figure 4 shows a typical plane truss 
element " $i, j "$. By treating the element as a simple structure with four degrees of freedom, the element stiffness matrix can be found. To obtain the element stiffness matrix the force system required to maintain unit displacements $\left(\boldsymbol{\delta}_{\mathrm{ij}}=\mathbf{1}\right.$ or $\left.\boldsymbol{\delta}_{\mathrm{ji}}=\mathbf{1}\right)$ at each freedom in turn must be evaluated.
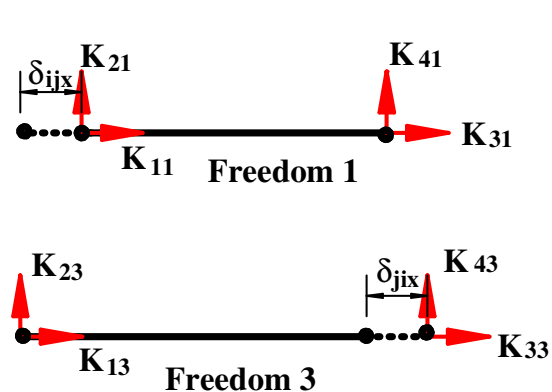

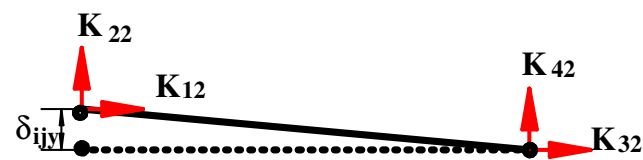

Freedom 2

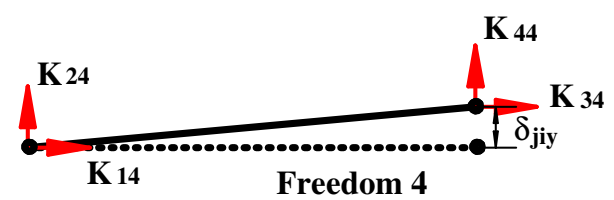

Figure 4: Evaluation of stiffness terms

For a plane truss or pin jointed member, only axial forces are involved and the stiffness terms can be found by inspection of Figure 4. All other stiffness coefficients are zero except;

$\boldsymbol{k}_{11}=k_{33}=\frac{E A}{L}$ and $k_{13}=k_{31}=-\frac{E A}{L}$, hence

$$
\left[\begin{array}{l}
q_{i j x} \\
q_{i j y} \\
q_{j i x} \\
q_{j i y}
\end{array}\right]=\left[\begin{array}{cccc}
\frac{A E}{L} & 0 & -\frac{A E}{L} & 0 \\
0 & 0 & 0 & 0 \\
-\frac{A E}{L} & 0 & \frac{A E}{L} & 0 \\
0 & 0 & 0 & 0
\end{array}\right]\left[\begin{array}{l}
\delta_{i j x} \\
\delta_{i j y} \\
\delta_{j i x} \\
\delta_{j i y}
\end{array}\right] \ldots .(4)
$$

The subscripts for force and displacements can be decoded as follows. The first two subscripts identify the element by the nodes at its ends, with the first character indicating the end under consideration. The third subscript defines the direction of the force or displacement (in the element axes system). If Eqn. (4) is split into sub matrices then;

$$
\left[\begin{array}{l}
\boldsymbol{q}_{i j} \\
\boldsymbol{q}_{j i}
\end{array}\right]=\left[\begin{array}{ll}
\boldsymbol{k}_{i i} & \boldsymbol{k}_{i j} \\
\boldsymbol{k}_{j i} & \boldsymbol{k}_{j j}
\end{array}\right]\left[\begin{array}{l}
\boldsymbol{\delta}_{i j} \\
\boldsymbol{\delta}_{j i}
\end{array}\right]
$$

hence,

$$
q_{j i}=k_{j i} \delta_{i j}+k_{j j} \delta_{j i}
$$

and

$$
q_{i j}=k_{i i} \delta_{i j}+k_{i j} \delta_{j i}
$$

On introducing the stiffness values, and eliminating rows and columns with zero $\boldsymbol{k}_{s}$, the load displacement equations may be written in matrix form as:

$$
\left[\begin{array}{l}
q_{i j} \\
q_{j i}
\end{array}\right]=\frac{A E}{L}\left[\begin{array}{cc}
1 & -1 \\
-1 & 1
\end{array}\right]\left[\begin{array}{l}
\delta_{i j} \\
\delta_{j i}
\end{array}\right]
$$

Or

$$
q=k^{\prime} \delta
$$

where $k^{\prime}=\frac{A E}{L}\left[\begin{array}{rr}1 & -1 \\ -1 & 1\end{array}\right]$

The matrix, k', is called the member stiffness matrix, and it is of the same form for each member of the truss. The four elements that comprise it are called member stiffness influence coefficients, $\mathbf{k}_{\mathbf{i j}}$. Physically, $\mathbf{k}_{\mathbf{i j}}$ represents the force at joint $\mathbf{i}$ when a unit displacement is imposed only at joint $\mathbf{j}$.

The structure stiffness matrix $\mathbf{K}$ is determined by assembling the transformed stiffness matrix $\mathbf{k}$ ' for each element. Usually this is done upon specifying all nodes, elements and materials of the structure. The transformation is done using force and displacement transformation matrices as expresses in the following sections.

\subsection{Displacement and Force Transformation Matrices}

Since a truss is composed of many members (elements), it is deemed necessary to transform the member forces $\boldsymbol{q}$ and displacements $\boldsymbol{\delta}$ defined in element local coordinates to a global $\boldsymbol{x}, \boldsymbol{y}$ coordinate system for the entire truss. For consistence, $\boldsymbol{x}$ is taken to be positive to the right and $\boldsymbol{y}$ positive upward. The smallest angles between positive $\boldsymbol{x}, \boldsymbol{y}$ global axes and the positive $\boldsymbol{x}$ ' local axis will be defined as $\boldsymbol{\theta}_{\mathbf{x}}$ and $\boldsymbol{\theta}_{\mathbf{y}}$ as shown in Figure 5. The cosines of these angles will be used in the matrix analysis that follows. These will be identified as $\boldsymbol{\lambda}_{\mathbf{x}}$ $=\cos \theta_{x}$ and $\lambda_{y}=\cos \theta_{y}$. Numerical values for $\lambda_{x}$ 
and $\lambda_{y}$ can be generated by a computer when the coordinates of near end $\mathbf{i},\left(\mathbf{x}_{\mathbf{i}}, \mathbf{y}_{\mathbf{i}}\right)$ and far end $\mathbf{j},\left(\mathbf{x}_{\mathbf{j}}, \mathbf{y}_{\mathbf{j}}\right)$ of the member have been specified. For example, if

$$
\begin{gathered}
\lambda_{x}=\cos \theta_{x}=\frac{x_{j}-x_{i}}{L}=\frac{x_{j}-x_{i}}{\sqrt{\left(x_{j}-x_{i}\right)+\left(y_{j}-y_{i}\right)}} \\
\lambda_{y}=\cos \theta_{y}=\frac{y_{j}-y_{i}}{L}=\frac{y_{j}-y_{i}}{\sqrt{\left(x_{j}-x_{i}\right)+\left(y_{j}-y_{i}\right)}}
\end{gathered}
$$

member $\mathbf{i j}$ of the truss shown in Fig. 5 has coordinates $\left(\mathbf{x}_{\mathbf{i}}, \mathbf{y}_{\mathbf{i}}\right)$ and $\left(\mathbf{x}_{\mathbf{j}}, \mathbf{y}_{\mathbf{j}}\right)$, then the direction cosines are found by Eqns (9) and (10); thus;

For easy handling of the analysis, the origin of the structure has to be located at a point where the $\boldsymbol{x}, \boldsymbol{y}$ coordinates of all the nodes will be positive.

\subsubsection{Displacement Transformation Matrix}

In global coordinates each end of the member can have two degrees of freedom or independent displacements (ref Figure 5); namely, joint $\mathbf{i}$ has $\Delta_{\mathbf{i j x}}$ and $\Delta_{\mathrm{ijy}}$, and joint $\mathbf{j}$ has $\Delta_{\mathrm{jix}}$ and $\Delta_{\mathrm{jiy}}$. Considering these displacements along the member; it is found that when the far end is held pinned and the near end is given a displacement of $\Delta_{\mathrm{ijx}}$, (Fig. 5), the

corresponding displacement (deformation) along the bar is $\Delta_{\mathrm{ijx}} \cos \theta_{\mathrm{x}}$. Likewise, a displacement $\Delta_{\mathrm{jiy}}$ will cause the bar to be displaced $\Delta_{\mathrm{jiy}} \cos \theta_{\mathrm{y}}$ along the x' axis. The effect of both global displacements is therefore

$$
\delta_{i j}=\Delta_{i j x} \cos \theta_{x}+\Delta_{i j y} \cos \theta_{y}
$$

In a similar manner, positive displacements $\Delta_{\mathrm{ijx}}$ and $\Delta_{\text {jiy }}$ successively applied at the far end $\mathbf{j}$, while the near end is held pinned, Figure 5, will cause the member to be displaced;

$$
\delta_{j i}=\Delta_{j i x} \cos \theta_{x}+\Delta_{j i y} \cos \theta_{y}
$$
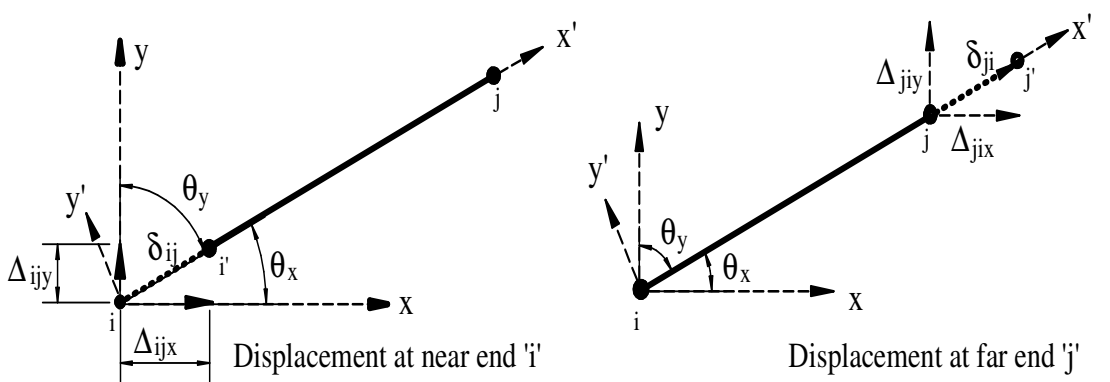

Figure 5: Global and local displacements

Letting $\lambda_{\mathrm{x}}=\cos \boldsymbol{\theta}_{\mathrm{x}}$ and $\boldsymbol{\lambda}_{\mathrm{y}}=\cos \boldsymbol{\theta}_{\mathrm{y}}$ represent the direction cosines for the member, it follows that:

$\delta_{i j}=\Delta_{i j x} \lambda_{x}+\Delta_{i j y} \lambda_{y}$

$\delta_{j i}=\Delta_{j i x} \lambda_{x}+\Delta_{j i y} \lambda_{y}$

which can be written in matrix form as;

$\left[\begin{array}{c}\delta_{i j} \\ \delta_{j i}\end{array}\right]=\left[\begin{array}{cccc}\lambda_{x} & \lambda_{y} & 0 & 0 \\ 0 & 0 & \lambda_{x} & \lambda_{y}\end{array}\right]\left[\begin{array}{c}\Delta_{i j x} \\ \Delta_{i j y} \\ \Delta_{j i x} \\ \Delta_{j i y}\end{array}\right]$

Or

$$
\delta=\mathbf{T} \Delta
$$

where

$$
T=\left[\begin{array}{cccc}
\lambda_{x} & \lambda_{y} & 0 & 0 \\
0 & 0 & \lambda_{x} & \lambda_{y}
\end{array}\right]
$$

From the above derivation., $\boldsymbol{T}$ transforms the four global $\boldsymbol{x}, \boldsymbol{y}$ displacements $\boldsymbol{\Delta}$ into the two local $\boldsymbol{x}$, displacements $\boldsymbol{\delta}$. Hence, $\boldsymbol{T}$ is referred to as the displacement transformation matrix.

\subsubsection{Force Transformation Matrix}

If a force is applied at the near end of the member, the far end held pinned, Fig. 6, here the global force components of $\boldsymbol{q}_{i j}$ at $\boldsymbol{i}$ are given by;

$Q_{i j x}=q_{i j} \cos \theta_{x}$

$Q_{i j y}=q_{i j} \cos \theta_{y}$

Likewise, if $\boldsymbol{q}_{j}$ is applied to the far, Fig. 6, the global force components at $j$ are

$$
\begin{aligned}
& Q_{j i y}=q_{j i} \cos \theta_{y} \\
& Q_{j i x}=q_{j i} \cos \theta_{x}
\end{aligned}
$$




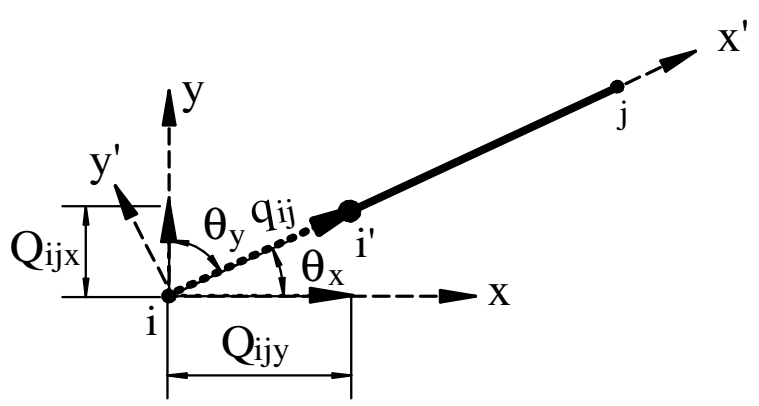

Load at near end 'i'

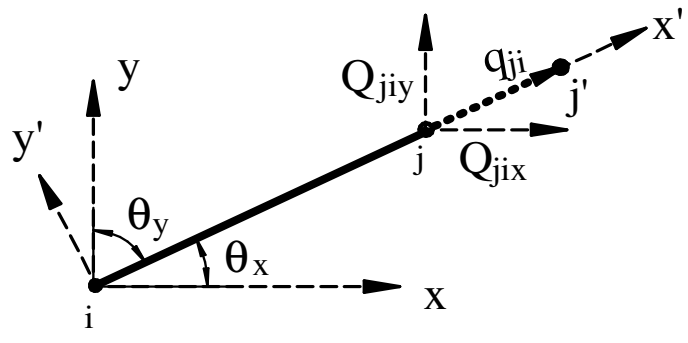

Load at far end ' $\mathrm{j}$ '

Figure 6: Loads in local and global coordinates

Using the direction cosines $\lambda_{\mathrm{x}}=\cos \theta_{\mathrm{x}}$ and $\lambda_{\mathrm{y}}=\cos$ $\boldsymbol{\theta}_{\mathbf{y}}$, these equations become

$$
\begin{array}{ll}
Q_{i j x}=q_{i j} \lambda_{x}, & Q_{i j y}=q_{i j} \lambda_{y} \\
Q_{j i x}=q_{j i} \lambda_{x}, & Q_{j i y}=q_{j i} \lambda_{y}
\end{array}
$$

In matrix form, the equations are presented as;

$$
\left[\begin{array}{c}
\frac{Q_{i j x}}{Q_{i j y}} \\
Q_{j i x} \\
Q_{j i y}
\end{array}\right]=\left[\begin{array}{cc}
\lambda_{x} & 0 \\
\lambda_{y} & 0 \\
0 & \lambda_{x} \\
0 & \lambda_{y}
\end{array}\right]\left[\begin{array}{l}
q_{i j} \\
q_{j i}
\end{array}\right]
$$

or

$$
\begin{aligned}
Q & =T^{T} q \\
\text { where } T^{T} & =\left[\begin{array}{cc}
\lambda_{x} & 0 \\
\lambda_{y} & 0 \\
0 & \lambda_{x} \\
0 & \lambda_{y}
\end{array}\right]
\end{aligned}
$$

In this case $\boldsymbol{T}^{T}$ transforms the two local ( $\mathbf{x}^{\mathbf{9}}$ ) forces $\mathbf{q}$ acting at the ends of the member into the four global $(\boldsymbol{x}, \boldsymbol{y})$ force components $\mathbf{Q}$. By comparison, this force transformation matrix is the transpose of the displacement transformation matrix, given by Eqn (13).

\subsection{Member Global Stiffness Matrix}

The member global stiffness matrix is obtained by appropriate relating the member's global force components $\mathbf{Q}$ to their respective global displacements $\boldsymbol{\Delta}$. On substituting Eqn.(12) into Eqn.(7), the member's forces $\mathbf{q}$ can be determined in terms of the global displacements $\Delta$ at its end points, namely,

$q=k^{\prime} T \Delta$

Substituting Eqn.(17) into Eqn. (15) yields the final result,

$\mathrm{Q}=\mathrm{T}^{\mathrm{T}} \mathrm{k}^{\top} \mathrm{T} \Delta$

Or

$Q=k \Delta$

where

$k=T^{T} k^{\prime} T$

The matrix $\mathbf{k}$ is the member's stiffness matrix in global coordinates. Since $\mathbf{T}^{\mathbf{T}}, \mathbf{T}$, and $\mathbf{k}^{\mathbf{\prime}}$ are known, the matrix $\mathbf{k}$ becomes:

$k=\left[\begin{array}{cc}\lambda_{x} & 0 \\ \lambda_{y} & 0 \\ 0 & \lambda_{x} \\ 0 & \lambda_{y}\end{array}\right] \frac{A E}{L}\left[\begin{array}{rr}1 & -1 \\ -1 & 1\end{array}\right]\left[\begin{array}{cccc}\lambda_{x} & \lambda_{y} & 0 & 0 \\ 0 & 0 & \lambda_{x} & \lambda_{y}\end{array}\right]$

Performing the matrix operations, the results becomes;

$k=\frac{A E}{L}\left[\begin{array}{cccc}i_{x} & i_{y} & j_{x} & j_{y} \\ \lambda_{x}^{2} & \lambda_{x} \lambda_{y} & -\lambda_{x}^{2} & -\lambda_{x} \lambda_{y} \\ \lambda_{x} \lambda_{y} & \lambda_{y}^{2} & -\lambda_{x} \lambda_{y} & -\lambda_{y}^{2} \\ -\lambda_{x}^{2} & -\lambda_{x} \lambda_{y} & \lambda_{x}^{2} & \lambda_{x} \lambda_{y} \\ -\lambda_{x} \lambda_{y} & -\lambda_{y}^{2} & \lambda_{x} \lambda_{y} & \lambda_{y}^{2}\end{array}\right] \begin{gathered}i_{x} \\ i_{y} \\ j_{x} \\ j_{y}\end{gathered}$

....(20) 
The location of each element in this $4 \times 4$ symmetric matrix is referenced with each global degree of freedom associated with the near end $\mathbf{i}$, followed by the far end $\mathbf{j}$. This is indicated by the code number notation along the rows and columns, that is, $\mathbf{i}_{\mathbf{x}}, \mathbf{i}_{\mathbf{y}}$, $\mathbf{j}_{\mathbf{x}}$, and $\mathbf{j}_{\mathbf{y}}$. Like $\mathbf{k}$ ' here $\mathbf{k}$ represents the forcedisplacement relations for each member when the components of force and displacement at the ends of the member are in the global or x,y directions. Each of the terms in the matrix is therefore a stiffness influence coefficient $\mathbf{k}_{\mathrm{ij}}$ which represents the $\mathrm{x}$ or $\mathrm{y}$ force component at $\mathbf{i}$ needed to cause an associated unit $\mathrm{x}$ or $\mathrm{y}$ displacement component at $\mathbf{j}$. As a result, each column of the matrix represents the four force components developed at the ends of the members when one of the ends undergoes a unit displacement related to its matrix column. For example, a unit displacement, $\Delta_{\mathrm{ix}}=\mathbf{1}$, will cause the four force components on the member shown in the first column of the matrix.

\subsection{Nodal Equilibrium}

Figure 7(a) shows a simple triangulated framework, and figure 7(b) shows how it might be idealized.

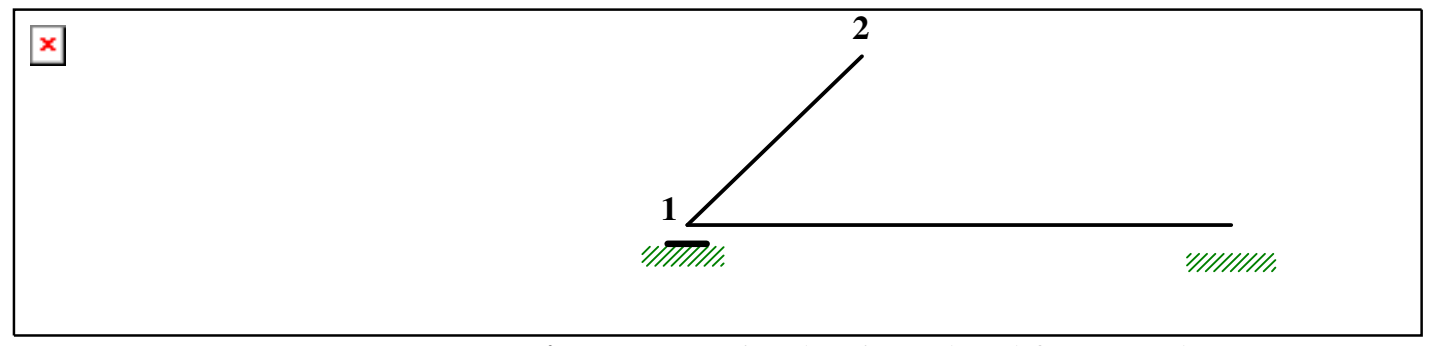

Figure 7: A simple triangulated framework

If node 2 is removed from the truss, its free body diagram is as shown in fig $8(a)$, and if the node is in equilibrium then there can be no net force acting upon it. Resolving each element force into components as shown in Figure 8(b), and summing the horizontal and vertical forces acting on the node produces the following equations:

$P_{2 x}+Q^{\prime}{ }_{21 x}+Q^{\prime}{ }_{23 x}+Q^{\prime}{ }_{24 x}+Q^{\prime}{ }_{25 x}=0$

and

$$
P_{2 y}+Q_{21 y}^{\prime}+Q_{23 y}^{\prime}+Q_{24 y}^{\prime}+Q_{25 y}^{\prime}=0
$$

or $\boldsymbol{P}_{2}+\boldsymbol{q}_{21}^{\prime}+\boldsymbol{q}_{23}^{\prime}+\boldsymbol{q}_{24}^{\prime}+\boldsymbol{q}_{25}^{\prime}=0$ where

$$
\boldsymbol{P}_{2}=\left[\begin{array}{l}
\boldsymbol{P}_{2 x} \\
\boldsymbol{P}_{2 y}
\end{array}\right], \quad \boldsymbol{q}_{21}^{\prime}=\left[\begin{array}{l}
Q^{\prime}{ }_{21 x} \\
Q^{\prime}{ }_{21 y}
\end{array}\right], \quad \cdots \quad \boldsymbol{q}_{25}^{\prime}=\left[\begin{array}{l}
Q^{\prime}{ }_{25 x} \\
Q^{\prime}{ }_{25 y}
\end{array}\right]
$$

(a)

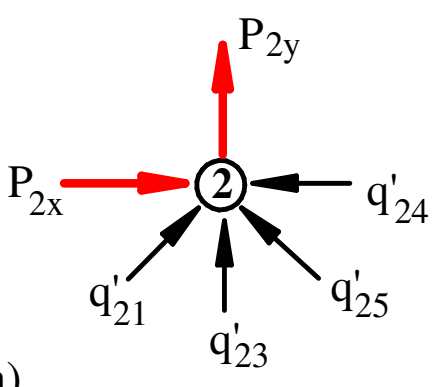

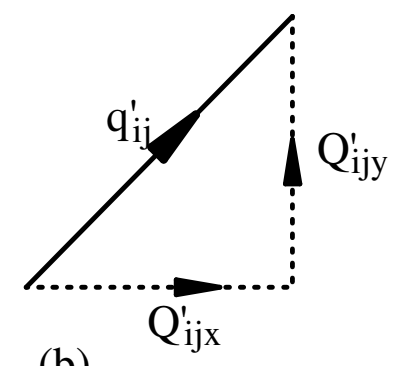

(b)

Figure 8: Free body diagram for node 2 
Hence, the equilibrium equation for node "i", which has elements "i,a", "i,b", "i,c",.... "i,n" framing into it, is

$$
P_{i}+q_{i a}^{\prime}+q_{i b}^{\prime}+q_{i c}^{\prime}+\cdots+q_{i n}^{\prime}=0
$$

One of the fundamental principles of static

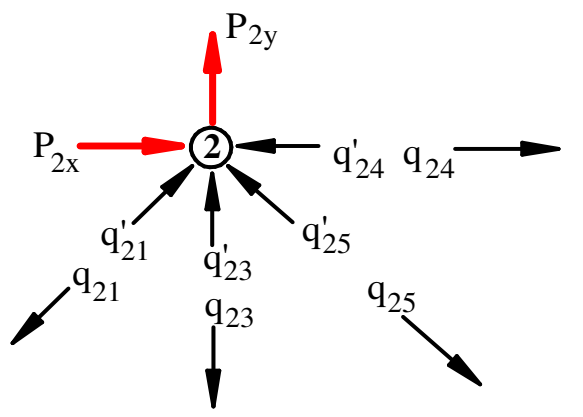

$$
\begin{array}{ll}
q_{21}=-q^{\prime}{ }_{21} & q_{23}=-q^{\prime}{ }_{23} \\
q_{24}=-q^{\prime}{ }_{24} & q_{25}=-q^{\prime}{ }_{25}
\end{array}
$$

Expressing the forces as components in the global axes system,

$$
\begin{array}{ll}
Q_{21 x}=-Q_{21 x}^{\prime} & Q_{25 x}=-Q_{25 x}^{\prime} \\
Q_{21 y}=-Q_{21 y}^{\prime} & Q_{25 y}=-Q_{25 y}^{\prime}
\end{array}
$$

Figure 9: Nodal forces and element end forces

and the equation of equilibrium for node 2 becomes

$P_{2}=q_{21}+q_{23}+q_{24}+q_{25}$

For node "i" with elements "i,a", "i,b", "i,c",.... "i,n" framing into it, the above equation becomes:

$$
P_{i}=q_{i a}+q_{i b}+q_{i c}+\cdots+q_{i n}
$$

The above equation simply states that the external applied load vector $\boldsymbol{P}_{\boldsymbol{i}}$ at node "i" must be balanced by the vectors of internal element end forces $\boldsymbol{q}_{i c}, \boldsymbol{q}_{i b}$, $\boldsymbol{q}_{i c}, \ldots \ldots, \boldsymbol{q}_{i n}$.

\subsection{Structure Stiffness Matrix}

Once all the member stiffness matrices are formed in global coordinates, it becomes necessary to assemble them in the proper order so that the structure stiffness matrix $\mathbf{K}$ for the truss can be found. This process of combining the member matrices depends on careful identification of the elements in each matrix. This is done by designating the rows and columns of the matrix by the four code numbers, $\mathbf{i}_{\mathbf{x}}, \mathbf{i}_{\mathbf{y}}, \mathbf{j}_{\mathbf{x}}, \mathbf{j}_{\mathbf{y}}$ used to identify the two global degrees of freedom that can occur at each end of the member. (see Eqn.20). The structure stiffness matrix will then have an order that will be equal to the highest code number assigned to the structure, since this represents the total number of degrees of freedom for the structure. When the $\mathbf{k}$ matrices are assembled, each element in $\mathbf{k}$ will then be placed in its same row and column designation in the structure stiffness matrix $\mathbf{K}$. In particular, when two or more members are connected to the same joint or node, then some of the elements of each of the $\mathbf{k}$ matrices will be assigned to the same position in the $\mathbf{K}$ matrix. When this occurs, the elements assigned to the common location must be added together algebraically. The reason for this becomes clear if one realizes that each element of the $\mathbf{k}$ matrix represents the resistance of the member to an applied force at its end. In this way, adding these resistances in the $\mathrm{x}$ or $\mathrm{y}$ direction when forming the $\mathbf{K}$ matrix is symbolic of determining the total resistance of each joint to a unit displacement in the $\mathrm{x}$ or $\mathrm{y}$ direction.

\subsection{Application of the Stiffness Method in the Truss Analysis}

Once the truss stiffness matrix, $\mathbf{K}$, is formed using the methods of the preceding section, it can then be used to determine the joint displacements, internal member forces and external force reactions. The loading vector $\mathbf{Q}$ has to be established according to the external global nodal loads. Also the displacement vector $\Delta$ has to be established, the known displacements are at the restrained nodes or supports and all others are unknown. Recalling Eqn.(18),

$\mathrm{Q}=\mathrm{K} \Delta$

For the first case, the load vector due to external loads $\mathbf{Q}_{\mathbf{k}}$ together with matrix $\mathbf{K}$ are computed to produce all the unknown displacements, $\Delta_{\mathbf{u}}$. In program TRAP, this has been achieved by using the Gauss elimination procedure.

$$
Q_{k}=K \cdot \Delta_{u}
$$


The second case, involves the determination of the internal forces for each element utilizing the already found displacements. The member forces can be determined using Eqn. (17), in which the contribution of each global displacement to the axial force of the element is taken into account. The said Eqn (17) is again given by,

$\mathrm{q}=\mathrm{k}^{\prime} \mathrm{T} \Delta$

Expanding this equation yields

$\left[\begin{array}{l}q_{i j} \\ q_{j i}\end{array}\right]=\frac{A E}{L}\left[\begin{array}{rr}1 & -1 \\ -1 & 1\end{array}\right]\left[\begin{array}{cccc}\lambda_{x} & \lambda_{x} & 0 & 0 \\ 0 & 0 & \lambda_{x} & \pi_{y}\end{array}\right]\left[\begin{array}{c}\Delta_{i j x} \\ \Delta_{i j y} \\ \Delta_{j i x} \\ \Delta_{j i y}\end{array}\right] \ldots$.

Since $\mathbf{q}_{\mathbf{i}}=\mathbf{-} \mathbf{q}_{\mathbf{j}}$ for equilibrium, only one of the forces may be determined. In this case, here only $\mathbf{q}_{\mathbf{j}}$ which is assumed to be in tension will be determined, Figure 6. Thus,

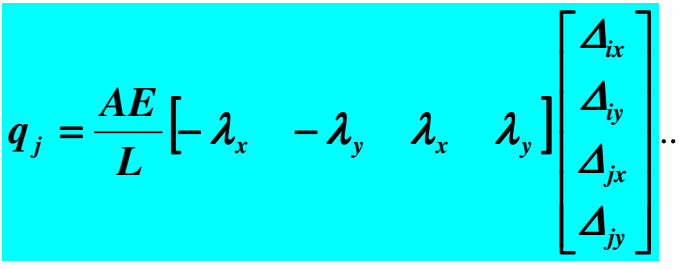

In particular, if the computed result using this equation is negative, the member is then in compression.

\section{Support Reactions}

The support reactions are computed at each node where the restraint is not equal to zero in a particular global direction using equation (24), thus:

$$
\begin{aligned}
& \boldsymbol{R}_{i x}=\lambda_{x} \cdot q_{i} \\
& \boldsymbol{R}_{i y}=\lambda_{y} \cdot q_{i}
\end{aligned}
$$

Normally, the reaction at a restrained node in any direction, say in $\mathbf{y}$-direction, $\mathbf{R}_{\mathbf{y}}$, is the summation of the contribution of each element force that joins at the specified node.

\subsection{THE PROGRAM TRAP}

The TRAP program has been prepared step by step using the stiffness method of analysis procedure as described in the previous sections. The programming software was Visual Studio 6.0 Professional [4]. The steps involved in the use of the program are;-

- Problem definition or truss data in general

- Identification of nodes

- Materials specifications
- Identification of elements

- Restraints introduction

- Global nodal loads introduction

Upon entering all the above data, the programme can analyse the structure and give the outputs which include nodal displacements and element axial forces. Also it has an option to output the plot of the original truss shape showing all nodes and the support points. In case the configuration of the structure is wrong, the user is advised to carefully check the input file item by item for corrections, then rerun the program. Details on preparation of the input data file is given in the Annex.

\subsection{Basic Truss Data}

The initial input data required include number of nodes, number of elements, number of materials, number of supports joints and number of loaded nodes. The program reads this input data from an input file in sequence as follows:

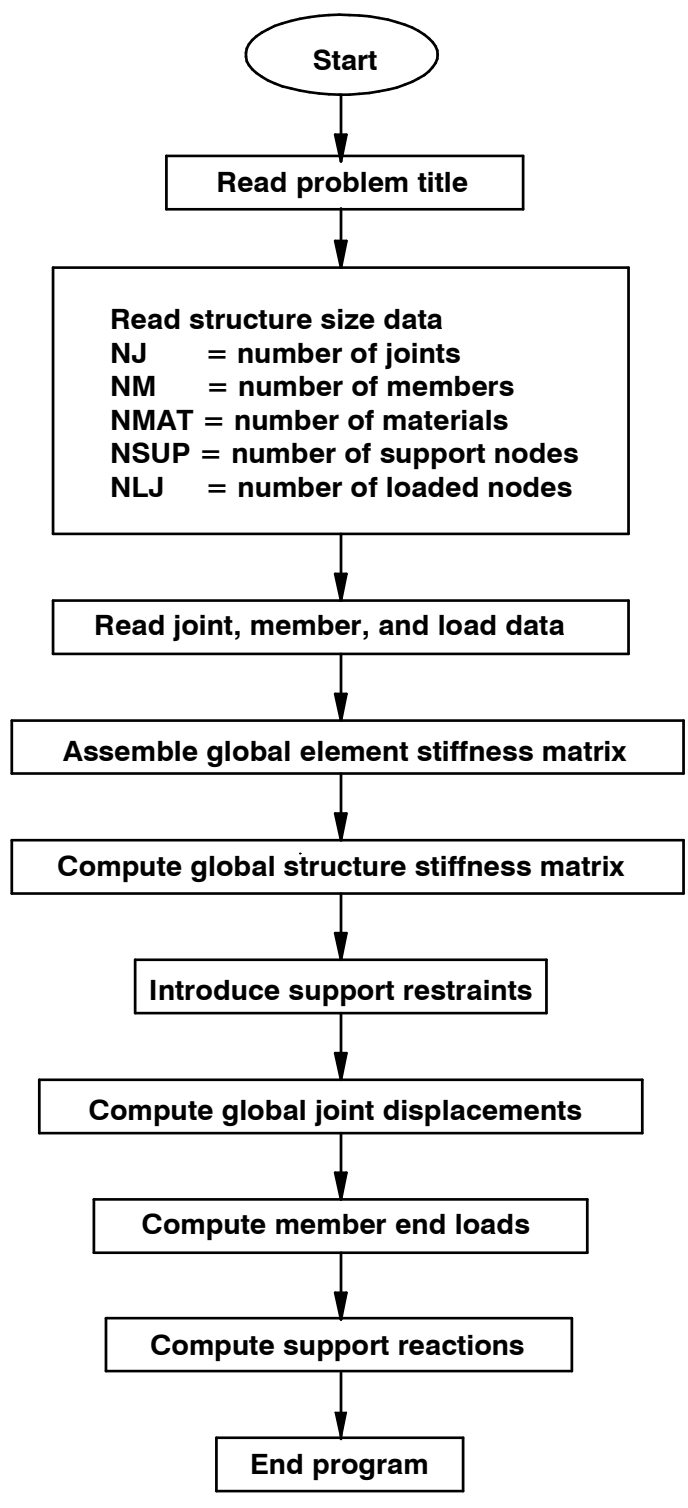




\section{$J, M, M A T, S U P, L J$;}

in which $\mathrm{J}$ is the number of nodes, $\mathrm{M}$ is the number of elements, MAT is the number of materials, SUP is the number of support joints and $\mathrm{LJ}$ is the number of loaded joints.

This is followed by an identification of the nodes by numbering them in a way that will lead to smaller half band width, $\boldsymbol{B A N D}$, of the structure stiffness matrix $\boldsymbol{K}$. The numbering is done as illustrated in Figure 10(a) and 10(b). The band width is given by the expression;

\section{BAND $=\operatorname{maximum}((\mathrm{J} 2-\mathrm{J} 1)+1) * 2$}

Where $\boldsymbol{J 1}$ is the near end and $\boldsymbol{J} \boldsymbol{2}$ is the far end of a particular element.

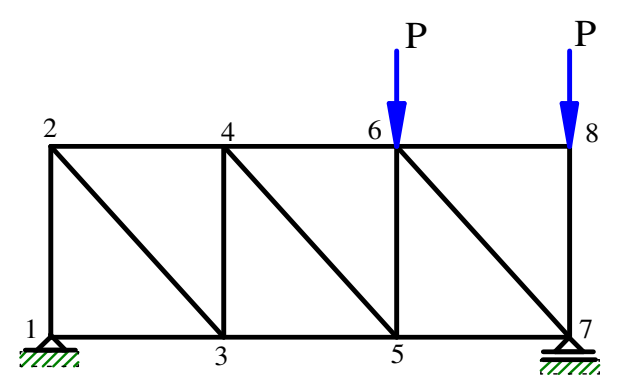

(a) Small BAND width

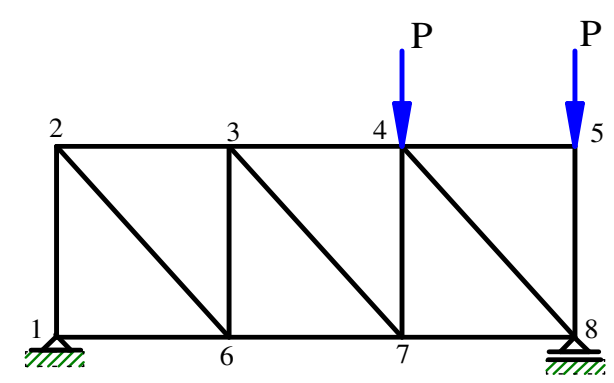

(b) Bigger BAND width

Figure 10: Nodes identification

For each element, the area, moment of inertia and modulus of elasticity have to be specified. Each set of such parameters has been classified as one type of material. Therefore one can have a number of types of materials in one truss. For example;

Let Types of materials be $=2$

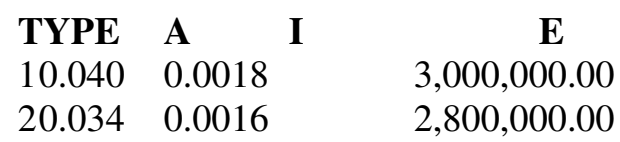

When the material type is 1 , the program will take Area $=0.04 \mathrm{~m}^{2}, \mathrm{I}=0.0018 \mathrm{~m}^{4}$ and $\mathrm{E}=3,000,000 \mathrm{~N} / \mathrm{m}^{2}$, while for type 2, the program will take Area $=0.034 \mathrm{~m}^{2}, \mathrm{I}=0.0016 \mathrm{~m}^{4}$ and $\mathrm{E}=$ $2,800,000 \mathrm{~N} / \mathrm{m}^{2}$.

To each element, the start node $\mathbf{J 1}$ and the end node J2 must be identified. Hence, the input file at this section will appear as follows;

$\begin{array}{lllll} & \text { Elem. No. } & \text { J1 } & \text { J2 } & \text { Type, } \\ \text { e.g; } & 1 & 1 & 2 & 1\end{array}$

This form of data will enable the program to recognize the coordinates of each element ends from which lengths and direction cosines are calculated; thus

$\mathrm{XL}=\mathrm{X}(\mathrm{J} 2)-\mathrm{X}(\mathrm{J} 1)$

$\mathrm{YL}=\mathrm{Y}(\mathrm{J} 2)-\mathrm{X}(\mathrm{J} 1)$

The length $\mathrm{L}=\left(\mathrm{XL}^{2}+\mathrm{YL}^{2}\right)^{0.5}$
The direction cosines, $\lambda_{x}$ and $\lambda_{y}$, are calculated using the expressions;
$\lambda_{x}=\frac{X L}{L}$
and

$$
\lambda_{y}=\frac{Y L}{L}
$$

Plotting of the original shape of the truss utilizes the coordinate values mentioned above.

\subsection{Restrained Nodes}

The restrained nodes are joints where by displacements or translations are prevented. Normally, restrained nodes are referred to as support nodes. In each restrained node, the directions of restraints are indicated by either " 1 " for restrained or "0" for unrestrained direction. An example (see Figure 10a) for the said restraints is given as follows:

$\begin{array}{lll}\text { Node } & \text { Rest. in }-\mathbf{x} & \text { Rest. in }-\mathbf{y} \\ 1 & 1 & 1 \\ 7 & 0 & 1\end{array}$

The above table indicates that the restrained nodes are 1 and 7. Node 1 is restrained in both $x-$ and $y$ direction, while Node 7 is restrained in $y$-direction only. The displacements of restrained nodes are zero in the specified directions. These restrained nodes help the structure to be stable and so enable the solution to be found. For an unstable structure, there is no solution possible. For the restrained nodes, the displacement vector $\boldsymbol{\Delta}$ elements are zero in the respective directions, while those which are not restrained, the vector elements are unknown quantities which are solved by the program. 


\section{Stiffness Matrices}

There are two stiffness matrices namely element stiffness matrix, $\boldsymbol{K}_{e}$, and global structure stiffness matrix $\boldsymbol{K}$. Before determining any stiffness matrix, the matrices are initialized in which $\boldsymbol{K}_{\boldsymbol{e}}$ is a $4 \times 4$ matrix and the global structure stiffness matrix $\boldsymbol{K}$ has a number of rows equal to the number of equations, $\boldsymbol{N E Q}$, which is $2 \boldsymbol{x} \boldsymbol{N J}$ for trusses, and number of columns equal to half band width, $\boldsymbol{B A N D}$. Due to the symmetry properties of the element stiffness matrix, $\boldsymbol{K}_{\boldsymbol{e}}$, only the upper triangle has been defined, thus:

$$
\begin{array}{lr}
\text { let } \quad A O=\frac{A E}{L}, \text { then } & \\
K_{e}(1,1)=A O * \lambda_{x}^{2}, & K_{e}(1,2)=A O * \lambda_{y}^{2} \\
K_{e}(1,3)=-A O * \lambda_{x}^{2}, & K_{e}(1,4)=-A O * \lambda_{x} \lambda_{y} \\
K_{e}(2,2)=A O * \lambda_{y}^{2}, & K_{e}(2,3)=-A O * \lambda_{x} \lambda_{y} \\
K_{e}(2,4)=A O * \lambda_{y}^{2}, & K_{e}(3,3)=A O * \lambda_{x}^{2} \\
K_{e}(3,4)=A O * \lambda_{x} \lambda_{y}, & K_{e}(4,4)=A O * \lambda_{y}^{2}
\end{array}
$$

After determining the $\boldsymbol{K}_{e}$ for an element, it is then added to the global structure stiffness matrix, $\boldsymbol{K}$ at the appropriate locations. The process is repeated till all elements are finished. Having the $\boldsymbol{K}$ matrix and the displacement vector $\boldsymbol{\Delta}$ the global external nodal loads vector, $\mathbf{Q}$, must be prepared. This load vector has one column with number of rows equal to the number of equations, $N E Q$.

\subsection{External Loading To The Structure}

For the truss-type structure, loads are applied at the nodes only. Nodes at which the loads are to be applied have to be defined, after which the load components are applied at each predetermined node in the global directions. An example of the load application with reference to Figure 10(a), assuming that $\mathrm{P}=14 \mathrm{kN}$, is as follows:

$\begin{array}{lcc}\text { Node } & \text { X-Component } & \text { Y-Component } \\ 6 & 0 & -14 \\ 8 & 0 & -14\end{array}$

(-ve because $\mathbf{Y}$ is positive upward)
The units of force are free, therefore after running the program, the force outputs will be in the units which the user had determined while inputting the loads. A sample input file is partly shown in Figure 12.

\section{Solution}

Due to the known loads, the program solves for all the unknown displacements in global directions using the force - displacement relationship;

\section{$\mathrm{Q}=\mathrm{K} \cdot \Delta$}

The Gauss elimination procedure has been applied in which the $\boldsymbol{Q}$ matrix is augmented to the global structure stiffness matrix $\boldsymbol{K}$. The displacements are determined by forward elimination and back substitution. When all displacements in the global axes system are known, element end forces are then determined using Eqn.(23), after which reactions are calculated using Eqn.(24). Some examples of the program run are given below.

\subsection{Sample Run Of Program TRAP}

Below is a configuration of a truss that has been analysed using the TRAP program. In Figure 11, the inscribed numbers indicate the nodes positions. The downward arrows are indicating the point loads in which all nodes in the top chord are loaded each with a $4.3 \mathrm{kN}$ down ward force. The support points are nodes 1, 16 and 17. Node 1 is restrained in both $\mathrm{X}$ and $\mathrm{Y}$ directions, while nodes 16 and 17 are restrained in X-direction only. The total number of nodes is 17 and the number of members is 31 . Much more data on the truss is given in the input file in the next section. The results are given in array form and in graphic form in Figures 12, 13 and 14. Figure 15 shows a help file displayed in a separate interface which is used as a guide when using the TRAP program. The given example is one the Authors design projects in which trusses of $12.0 \mathrm{~m}$ span were analysed for KKKT -Sinza in Dar es Salaam. 


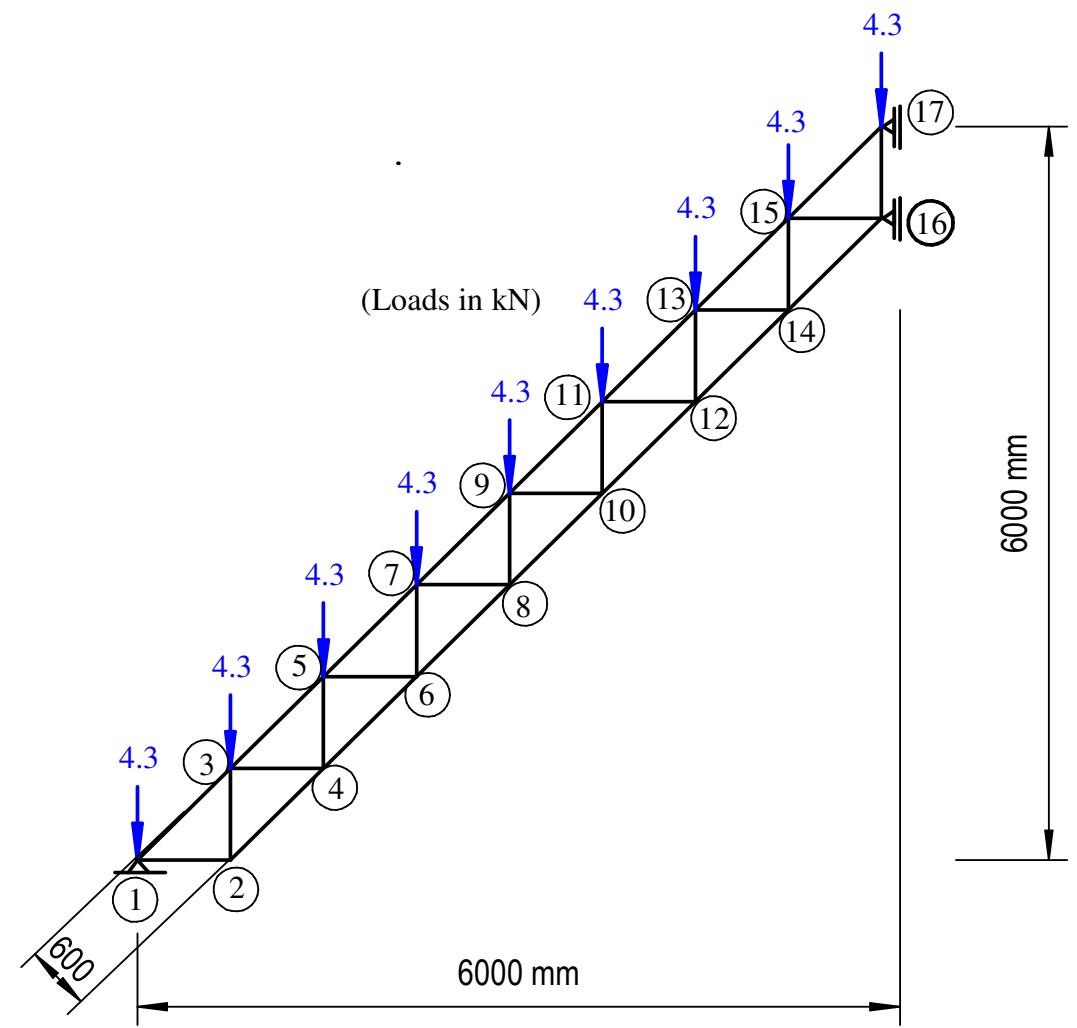

Figure 11: Sample analyzed frame

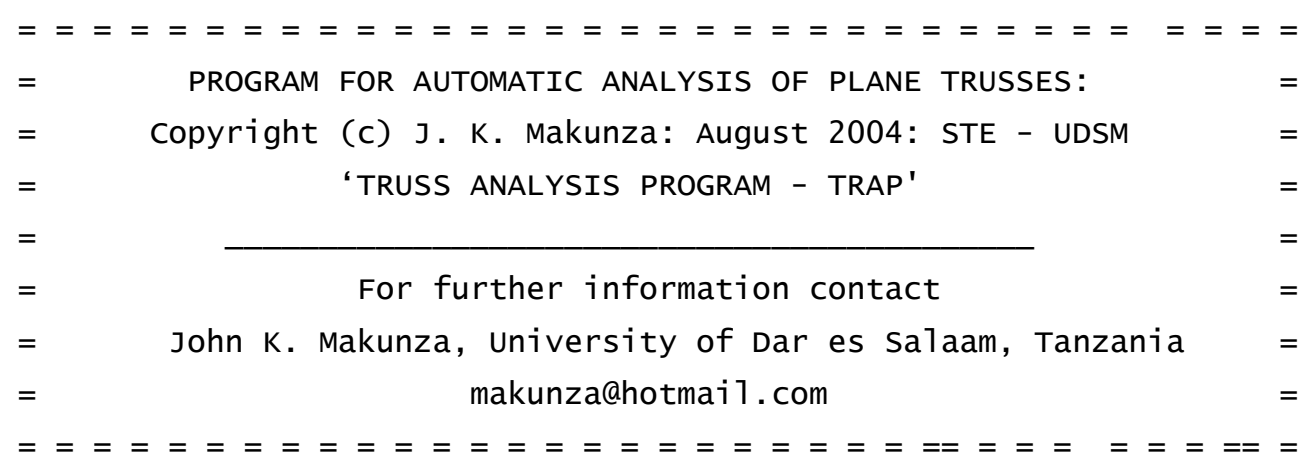

P R O G R A M R U N

O N D A Y A N D T I M E $=12 / 6 / 2006$ 6:03:42 PM

PLANE FRAME ANALYSIS

NUMBER OF JOINTS 17

NUMBER OF MEMBERS 31

NUM8ER OF MATERIALS 1

NUM8ER OF SUPPORT JOINTS 3

NUMBER OF LOADED JOINTS 9

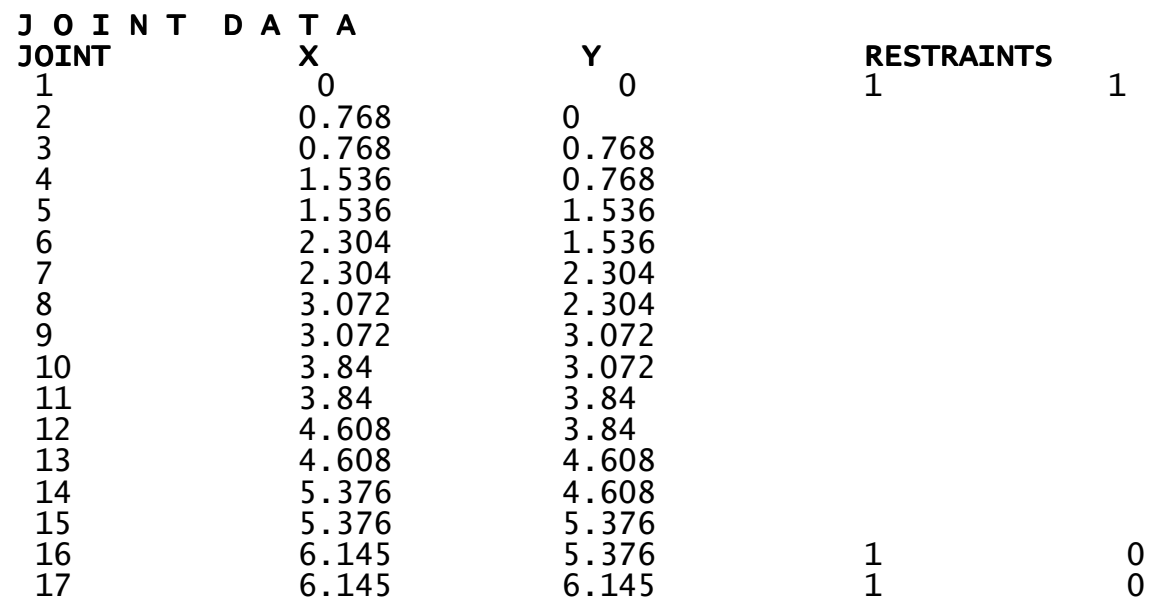


M A T E R I A L D A T A

No.

Area

I-Mom

E-Mod

1

0.05

0.0044

210000000

M E M B E R D A T A

$\begin{array}{cccc}\text { MEMBER } & \text { J1 } & \text { J2 } & \text { TYPE } \\ 1 & 1 & 2 & 1 \\ 2 & 1 & 3 & 1 \\ 3 & 2 & 3 & 1 \\ 4 & 2 & 4 & 1 \\ 5 & 3 & 4 & 1 \\ 6 & 3 & 5 & 1 \\ 7 & 4 & 5 & 1 \\ 8 & 4 & 6 & 1 \\ 9 & 5 & 6 & 1 \\ 10 & 5 & 7 & 1 \\ 11 & 6 & 7 & 1 \\ 12 & 6 & 8 & 1 \\ 13 & 7 & 8 & 1 \\ 14 & 7 & 9 & 1 \\ 15 & 8 & 9 & 1 \\ 16 & 8 & 10 & 1 \\ 17 & 9 & 10 & 1 \\ 18 & 9 & 11 & 1 \\ 19 & 10 & 11 & 1 \\ 20 & 10 & 12 & 1 \\ 21 & 11 & 12 & 1 \\ 22 & 11 & 13 & 1 \\ 23 & 12 & 13 & 1 \\ 24 & 12 & 14 & 1 \\ 25 & 13 & 14 & 1 \\ 26 & 13 & 15 & 1 \\ 27 & 14 & 15 & 1 \\ 28 & 14 & 16 & 1 \\ 29 & 15 & 16 & 1 \\ 30 & 15 & 17 & 1 \\ 31 & 16 & 17 & 1 \\ & & & \end{array}$

J O I N T L O A D S

\begin{tabular}{ccc} 
JOINT & WX & WY \\
1 & 0 & -4.3 \\
3 & 0 & -4.3 \\
5 & 0 & -4.3 \\
7 & 0 & -4.3 \\
9 & 0 & -4.3 \\
11 & 0 & -4.3 \\
13 & 0 & -4.3 \\
15 & 0 & -4.3 \\
17 & 0 & -4.3 \\
\hline
\end{tabular}

NUMBER OF EQUATIONS $=34$

HALF BAND WIDTH $=6$

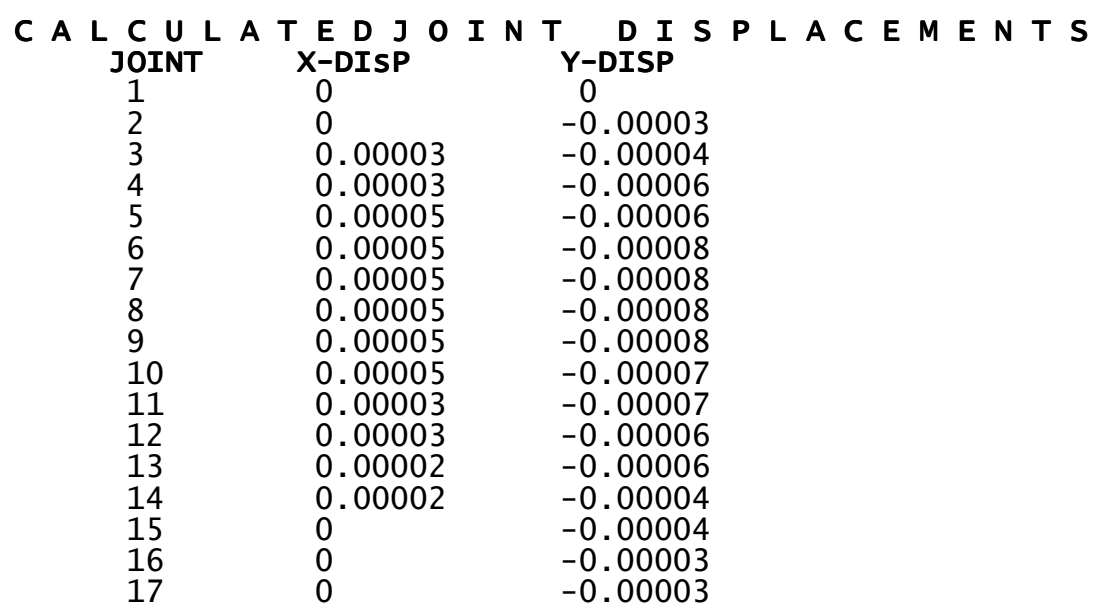




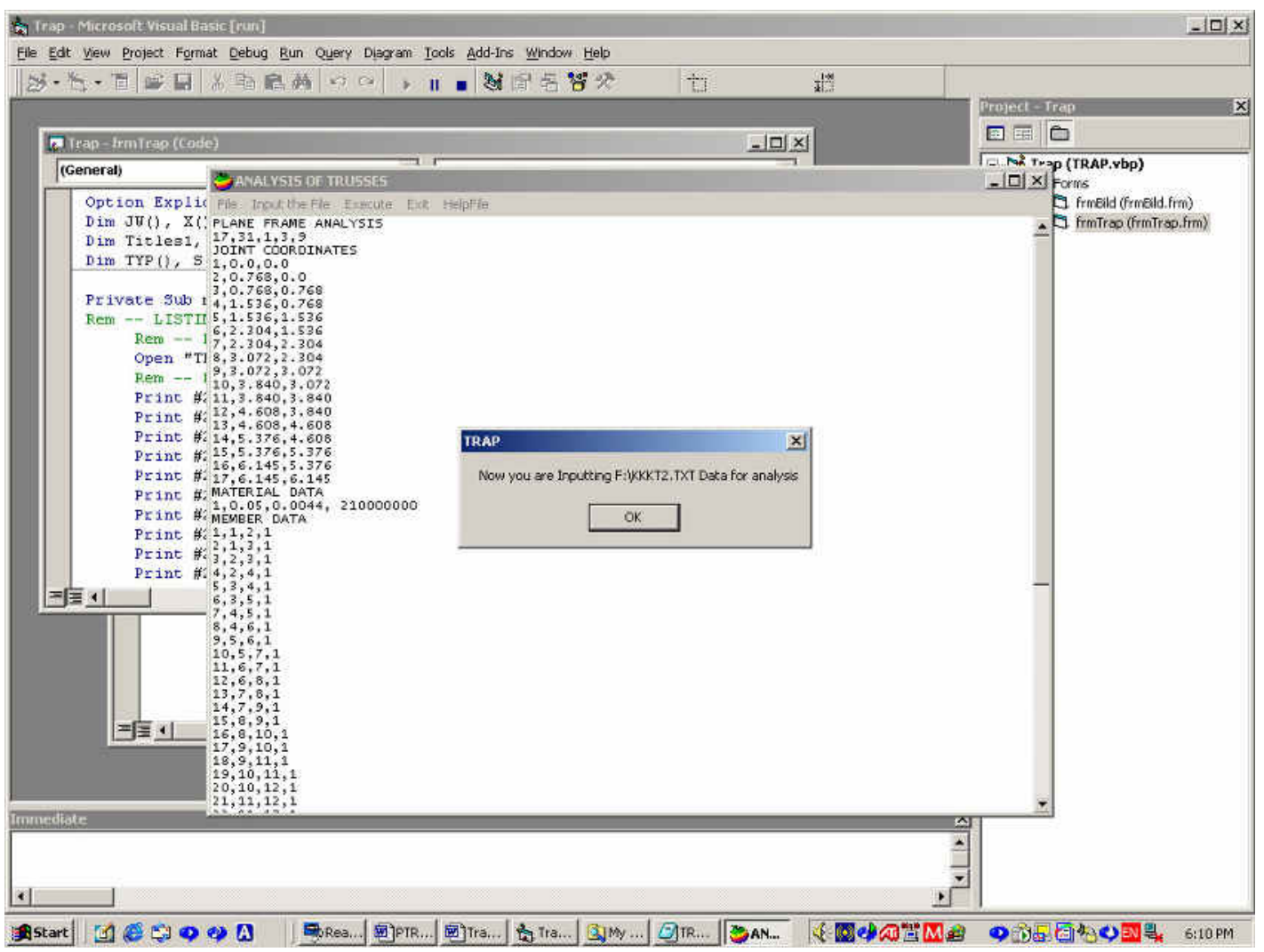

Figure 12: Inputting data to the program

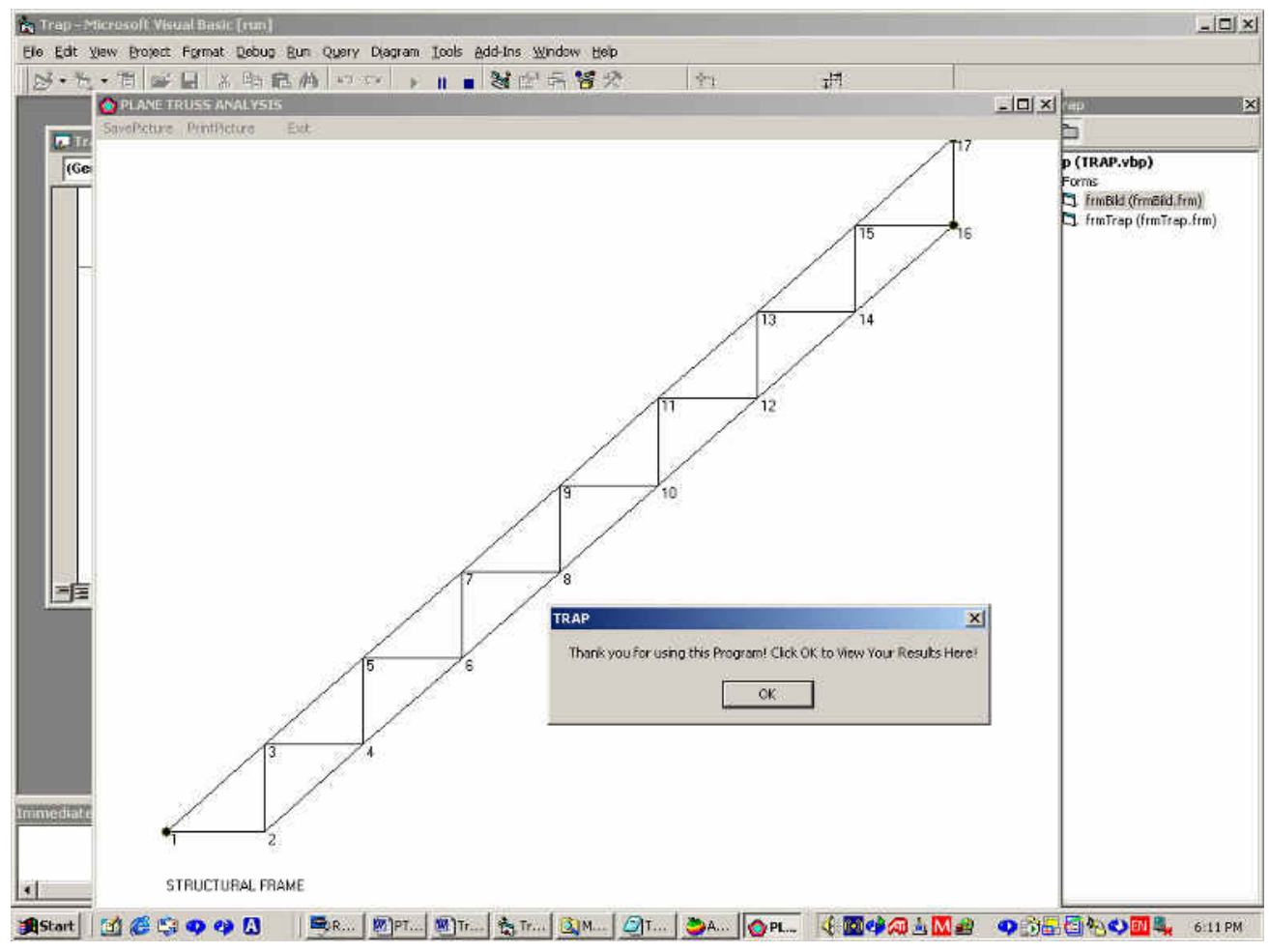

Figure 13: Plot output for the original shape of the truss 


\section{A L C U L A T E D E L E ME N T F OR C E S}

$\begin{array}{ccccc}\text { MEMBER } & \text { J1 } & \text { J2 } & \text { FORCE } & \text { LENGTH } \\ 1 & 1 & 2 & 8.94 & 0.768 \\ 2 & 1 & 3 & -48.65 & 1.086 \\ 3 & 2 & 3 & -8.94 & 0.768 \\ 4 & 2 & 4 & 12.64 & 1.086 \\ 5 & 3 & 4 & 4.64 & 0.768 \\ 6 & 3 & 5 & -55.21 & 1.086 \\ 7 & 4 & -4.64 & 0.768 \\ 8 & 4 & 6 & 19.2 & 1.086 \\ 9 & 5 & 7 & 0.34 & 0.768 \\ 10 & 5 & 7 & -55.69 & 1.086 \\ 11 & 6 & 8 & -0.34 & 0.768 \\ 12 & 6 & 8 & 19.68 & 1.086 \\ 13 & 7 & 9 & -3.96 & 0.768 \\ 14 & 7 & 9 & 3.96 & 1.086 \\ 15 & 8 & 10 & 14.08 & 0.768 \\ 16 & 8 & 10 & -8.26 & 1.086 \\ 17 & 9 & 11 & -38.41 & 0.768 \\ 18 & 9 & 11 & 8.26 & 1.086 \\ 19 & 10 & 12 & 2.4 & 0.768 \\ 20 & 10 & 12 & -12.56 & 1.086 \\ 21 & 11 & 13 & -20.65 & 0.768 \\ 22 & 11 & 13 & 12.56 & 1.086 \\ 23 & 12 & 14 & -15.36 & 0.768 \\ 24 & 12 & 14 & -16.86 & 1.086 \\ 25 & 13 & 15 & 3.2 & 0.768 \\ 26 & 13 & 15 & 16.82 & 1.086 \\ 27 & 14 & 16 & -39.18 & 1.768 \\ 28 & 14 & 16 & 33.087 \\ 29 & 15 & 17 & -27.69 & 0.769 \\ 30 & 15 & & & 1.088 \\ 31 & 16 & & 0.769\end{array}$

R E A C T I O N S

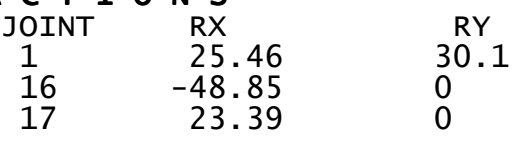

SOL U T I O N C O M P L E TE D A T T I ME $=6: 03: 48$ PM

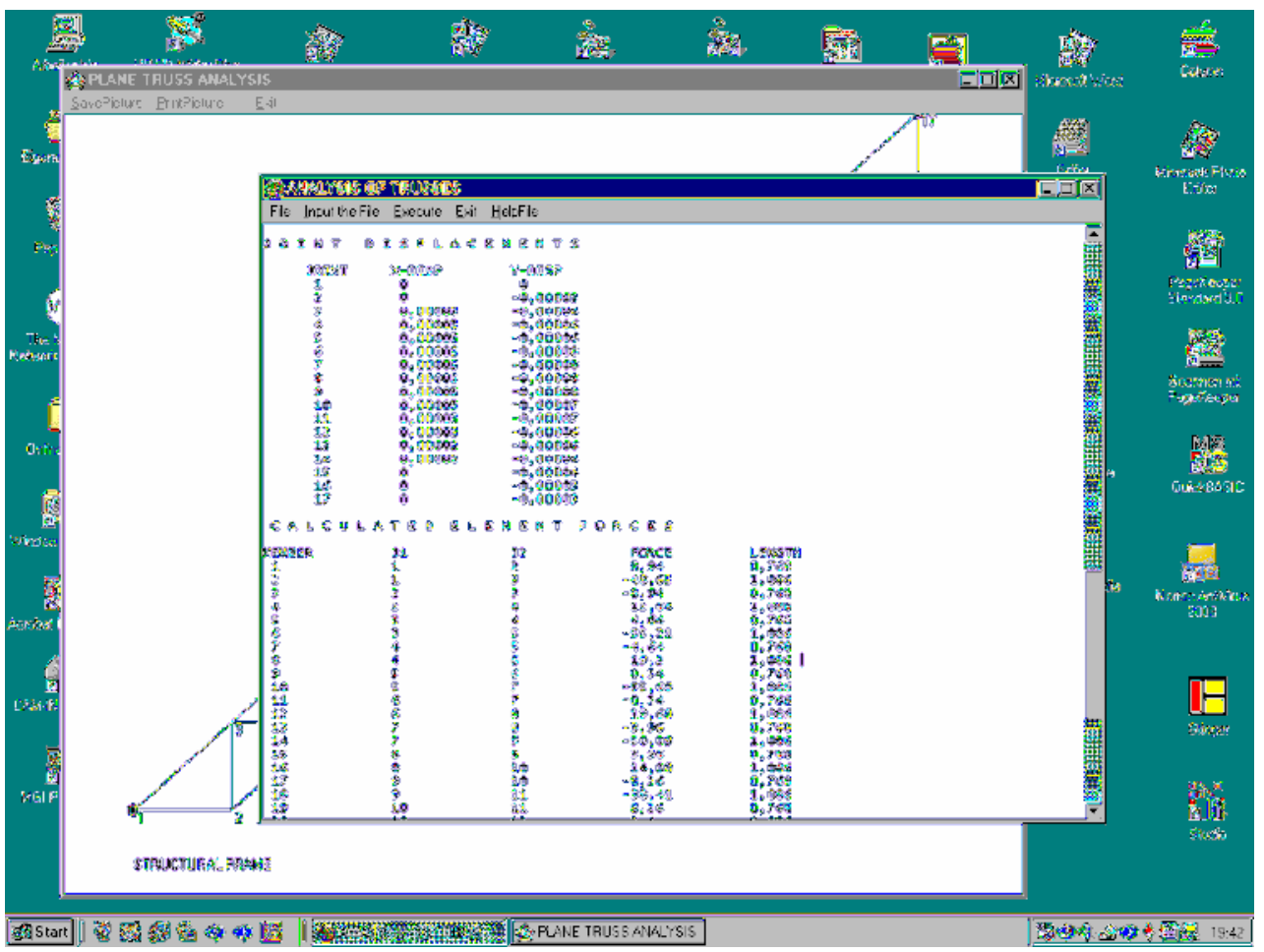

Figure 14: Output of element forces and lengths. 


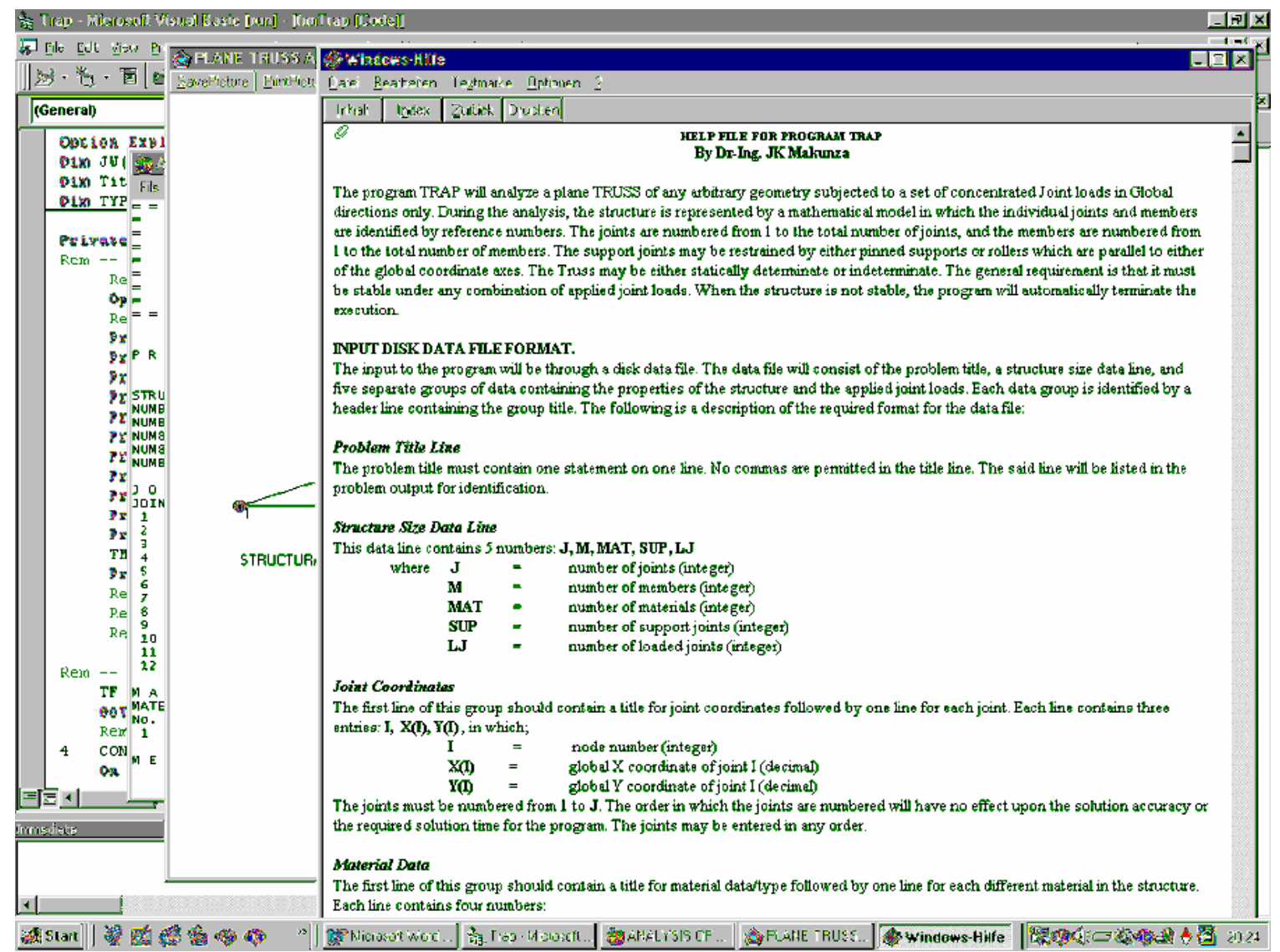

Figure 15: Help file for program TRAP

\subsection{DISCUSSION}

This example shows how the Program TRAP is efficient in the analysis of truss structures. The program can analyze any plane truss without limitations in nodes, restraints, and number of elements. The outputs are displacements and element axial forces in the units the user may choose. If the force is negative, it means it is compressive and when positive it means the force is tension. There is an option during the run-time from which one can opt to view the plot of the undeformed structure such as the one shown in Figure 13. There are two output windows, one for graphical plot and the other for numerical tabulated results.

After viewing the results, both numerical and graphical outputs, you may print to a printer or save each as a file. You may then highlight and copy the results to a word document.

\subsection{CONCLUSIONS AND RECOMMENDATION}

From the analysis and results obtained, it is concluded that the program TRAP:

* can be used to analyze plane trusses of any size or number of nodes and elements,
* is user friendly and solves analytical problems at higher speed with greater accuracy,

* outputs displacements, axial forces and plot of the original shape of the truss,

* can be installed in windows operating systems limited to Win95, Win98,

Win Me, Win2000 and Win XP.

It is therefore recommended that this program be used for analysis of plane trusses, as it analyses quickly with outputs which can be used for design purposes especially for timber and steel trusses. The program has to be further developed so as to increase the graphical outputs such as axial forces in each element and plot of the deflected shape of the analyzed truss.

This TRAP program is suitable for use during the analysis of PLANE TRUSSES of any form, after which the axial forces obtained can be used to design the respective members in either tension action or compression action. 


\subsection{REFERENCES}

Ghali, A., and Neville, A. M., (1989), "Structural Analysis, A Unified Classical and Matrix Approach, Chapman and Hall, London, ISBN 04122904008.

Statik im Bauwesen, Statisch unbestimmte ebene Systeme, Band 3, Bochmann, Verlag für Bauwesen, Berlin, 1995.
Visual Studio 6.0 Professional, Microsoft Visual Studio, 1998.

William, Weaver, J.R., and James, M. Gere, (2002), "Matrix Analysis of Framed Structures", $2^{\text {nd }}$ Edition, CBS Publishers, New Delhi, India,. 